\title{
Endoscopic procedures in ART: an overview
}

Published online: 4 March 2004

(C) Springer-Verlag Berlin / Heidelberg 2004

\begin{abstract}
In the past two decades, improvement in instrumentation has lead to widespread use of gynaecological endoscopy. Most doctors involved in delivering infertility services have had some training and experience in gynaecological endoscopy. Laparoscopy is a commonly performed endoscopic procedure in the evaluation and management of the infertile couple. The economic and safety issues of diagnostic laparoscopy should be considered. Evidence suggests that laparoscopy has a role to play in evaluating women with unexplained infertility prior to ART. Infertility investigations and treatment are a long process. A one-stop approach has therefore been proposed for couples presenting with infertility. Transvaginal hydrolaparoscopy is systematically combined with mini-hysteroscopy, chrompertubation, fimbrioscopy and salpingoscopy, if needed. The combination of these procedures is referred to as "fertiloscopy". This approach has been shown to be feasible, accurate for determining tubal patency (95\%), safer than laparoscopy with respect to bowel injuries, better tolerated and certainly costeffective.
\end{abstract}

Keywords Endoscopy $\cdot$ Infertility $\cdot$ Investigation

\section{Introduction}

In the past two decades, improvement in instrumentation has led to widespread use of gynaecological endoscopy. The ability to achieve haemostasis during endoscopic procedures has made it possible to perform surgical procedures via the laparoscope and hysteroscope.

Prior to the invention of electricity, endoscopy was practiced with the help of either candlelight (Bozzani 1805) or by reflecting light by mirrors. Nitze from

\footnotetext{
A. Sharma $(\bowtie) \cdot$ R. Bhalla $\cdot$ P. O’Donovan

Bradford Royal Infirmary,

The Merit Centre, Bradford, UK

e-mail: ajayssharma@hotmail.com

Tel.: +44-1274-366945

Fax: +44-1274-364888
}

Germany developed the lens system allowing magnification of the viewed area. Kelling also from Germany (1902) first reported peritoneal endoscopy in dogs creating a pneumoperitoneum using a needle. Later, Jacobeus (1910) used a trocar and cannula to induce pneumoperitoneum in women. Zollikoffer from Switzerland used carbon dioxide for the first time for creating a pneumoperitoneum. Fervers in Germany (1933) reported biopsy and cauterisation of intraabdominal adhesions at laparoscopy. Palmer of France was the first gynaecologist to use laparoscopy widely (1947). Hopkins and Kapany introduced fibre optics in 1952. Frangenheim in Germany (1963) and Palmer in France improved electrocoagulation for tubal sterilisation by laparoscopy. Kurt Semm in Germany continued to improve instruments for endoscopic surgery.

As early as 1000 A.D., Abulkassim used a mirror to reflect light into the vaginal vault. Desormeaux (1853) in France reported the first "satisfactory" hysteroscopy. Clado (1898) in France described several models of hysteroscope and described the technique in detail. A treatise on contact hysteroscopy by David followed this in 1908. Rubin in 1925 used carbon dioxide and Gauss (1928) used water to distend the uterine cavity. Lindemann (1970) further developed the use of carbon dioxide for uterine distension. With the advent of better optics and instrumentation, hysteroscopic techniques have become sophisticated and safe.

Refinements in instrumentation and techniques have led to worldwide acceptance of laparoscopy and hysteroscopy for diagnostic and operative procedures. Endos-

Table 1 Endoscopic procedures performed for ART

Pelvic cavity
Laparoscopy
Transvaginal hydrolaparoscopy
Uterine cavity
Hysteroscopy
Fallopian tubes
Falloposcopy
Salpingoscopy

Laparoscopy

Transvaginal hydrolaparoscopy

Terine cavity

Falloposcopy

Salpingoscopy 
Table 2 Laparoscopic procedures performed in ART

Diagnostic
Chrompertubation
Salpingoscopy
Therapeutic
Division of adhesions
Management of ovarian cysts
Management of endometriosis

copy and ART are inextricably linked (Table 1). Endoscopy plays a role both in diagnosis and management of subfertility. Most doctors involved in delivering infertility services have had some training and experience in gynaecological endoscopy.

\section{Laparoscopy}

Laparoscopy has been regarded as a gold standard in the evaluation of sub-fertile women (Table 2). It not only allows visualisation of the pelvic organs but also permits therapeutic procedures to be carried out at the same time. Being minimally invasive, it is well accepted by women. Tubal patency can be confirmed by chrompertubation, endometriosis can be identified, staged and treated at the same time, and adhesions can be divided if found at laparoscopy. Visualisation of the upper abdomen is another advantage; however, the function of the fallopian tube cannot be assessed at laparoscopy.

\section{Laparoscopic equipment and avoiding complications}

The position of the patient should be optimal to allow adequate access for placement of the trocars in the abdomen while allowing easy access to the vagina for uterine manipulation. A modified lithotomy position is adopted with the hips angled at $45^{\circ}$ and the knees draped over supports on either side. The bladder is emptied to pre-empt any injury to it during insertion of trocar.

Diagnostic laparoscopy should ideally be performed during the follicular phase of the menstrual function after cessation of the menses [1]. The peritoneum is the most adherent at the umbilicus making it the thinnest part of the abdominal wall. The Verres needle should therefore be inserted in the base of the umbilicus. It is also the most cosmetically acceptable. It is inserted initially vertically and then angled towards the uterus. The abdominal wall is pulled away from the great vessels by grasping the umbilicus with the thumb and the index finger. The Verres needle should be held halfway up its shaft to prevent inadvertent damage to major vessels due to precipitate insertion. Throughout the insertion of the Verres needle the patient should be kept horizontal, because only the horizontal plane provides reference to the underlying anatomy. The practice of tilting the patient in a Trendlenburg position before pneumoperitoneum is established is unsafe. Various techniques have been described to ensure correct placement of the needle in the peritoneal cavity. Five millilitres of saline is injected through the Verres needle. No aspirate should be obtained in the syringe if the needle is in the peritoneal cavity. The swinging needle test is not recommended as the tip of the Verres needle cannot be visualised during the procedure, and moving it without due care may damage the abdominal viscera. The insuffulation technique is widely practised and easy. Once the surgeon is satisfied that the Verres needle is in the peritoneal space, the gas flow is attached and insufflation is commenced. If the gas flow is at or more than $1 \mathrm{l} / \mathrm{min}$ and the intraabdominal pressure is less than $10 \mathrm{mmHg}$, then the needle is most probably in the peritoneal cavity. The trocar insertion is the most hazardous part of the procedure. The trocar insertion follows similar directions as the insertion of the Verres needle. The trocar should be aimed at the uterus and kept vertically in the midline as deviation to the left or right of the midline can result in damage to the pelvic vessels. Laparoscopic trocars are approximately $10 \mathrm{~cm}$ long and, if inserted to their full extent, can easily perforate bowel or vessels. A better option is a shorter trocar $(6-7 \mathrm{~cm}$ in length) with an atraumatic fixing mechanism such as a screw. The traditional valve mechanism found on many trocars restricts free movement on instruments within the trocar. Plastic sheaths are now available and are a suitable alternative. Secondary trocars are usually placed suprapubically or laterally. Insertion should be under direct vision. The inferior epigastric vessels can be avoided by direct inspection of the anterior abdominal wall. The superficial vessels can be observed at transillumination. With open laparoscopy as advocated by Hasson, the risk of injury to blood vessels is reduced; nevertheless, they still could occur especially in thin patients [2].

The most common laparoscopes used in gynaecological surgery are 5 or $10 \mathrm{~mm}$ in diameter. A $150-\mathrm{W}$ halogen or xenon light source is necessary for the optical system to function optimally. A thicker light cable $(10 \mathrm{~mm})$ transmits more light and is preferable. The light cable should be replaced if more than one-third of the fibres are broken. Although the light transmitted is termed "cold light", it can still generate significant heat if kept in contact with the patient or the drapes. The use of threechip cameras with the video system gives a more realistic picture of tissues and organs. Atraumatic grasper should be used to manipulate the ovaries and the fallopian tubes. Tissue cutting can be achieved using scissors, EndoGIA or laser. While EndoGIA would add to the cost of surgery, laser is not commonly available. Tissue haemostasis is achieved by monopolar or bipolar electrocautery. The current delivered to the tissue should be enough to desiccate the tissues and not too much to cause charring. Many surgeons combine monopolar cautery with scissors to allow coagulation of tissues immediately prior to cutting the tissues. The operative field is kept clear by the use of suction irrigation system. Small tissue specimens can be removed directly from the laparoscopic portals. Larger specimens may be retrieved by use of endobags or culdotomy. 
Management of adhesions

Adhesions are formed as a reaction to trauma from surgery, infection or endometriosis. Apart from distorting the anatomy, adhesions can also lead to pain, infertility or bowel obstruction. Laparotomy may lead to reformation of adhesions. This may be due to tissue handling, drying of tissues, blood clots and reaction to suture materials, ischaemia and instruments.

Laparoscopic treatment has several advantages. Apart from the magnification and reduced tissue drying, tissue handling is minimal. This leads to a reduction of adhesion formation. This has been confirmed in animal models and while comparing the effects of laparoscopy/laparotomy for treatment of ectopic pregnancy [3]. Laparoscopic adhesiolysis thus seems to a better option as compared with laparotomy. Insertion of the primary port may depend on previous abdominal surgery. At least two accessory portals are required. Adhesions are divided as far as possible from viable organs. Monopolar or bipolar diathermy with scissors is most frequently used. In order to minimise lateral tissue damage, it is best to diathermise with bipolar forceps prior to division with scissors. Traction on adhesions during division helps in dissection. Bleeding should be kept to minimum to reduce further adhesion formation. Bowel adhesions should be divided first so that it can be pushed away from the operative field. Freeing the ovaries and then the tubes follows this [4]. At the end of surgery, the pelvis is filled with solutions such as adept, which forms a film around the tissues and prevents recurrence of adhesions. Laparoscopic salpingovariolysis has the advantage of reduction in postoperative pain, quicker recovery and return to normal activities. Laparoscopic salpingostomy is frequently performed. Gross anatomy is first restored as far as possible. The fallopian tube is filled with methylene blue dye to dilate the ampullary portion. This identifies the thinnest part of the tubal wall. The distal part of the tube is stabilised with an atraumatic grasper. A cruciate incision is made on the fallopian tube using scissors. The flaps are retracted permanently by coagulating the serosa using a ball cautery.

Recently, investigators have demonstrated that laparoscopic salpingectomy or proximal tubal occlusion may enhance pregnancy rates of in vitro fertilisation in women with irreparable hydrosalpinges [5].

\section{Laparoscopic ovarian surgery}

Ovarian cysts are frequent findings at ultrasound during assisted reproductive treatment. It is important to exclude malignancy by history, examination, ultrasound with Doppler blood flow measurements and tumour markers. If malignancy can be confidently excluded, then laparoscopic treatment may be appropriate. Ovarian cystectomy should be performed with the aim of removing the cyst intact. The ovarian tissue overlying the cyst is incised using either scissors or monopolar diathermy. The inci- sion is preferred on the antimesenteric border of the ovary away from the blood vessels. Atraumatic graspers grasp the cyst wall and the overlying ovarian tissue is stripped off it using blunt or sharp dissection. Aqua dissection can be used to good effect. Invariably, the cyst wall ruptures during these manipulations. Bipolar diathermy may be required to stop any bleeding. The cyst wall could either be removed through the primary laparoscope portal or an endobag may be utilised. When enucleating endometriotic cysts, it may be impossible to dissect and remove the cyst wall. In such cases, the cyst lining can be destroyed by diathermy. Finally, the cyst cavity is irrigated and bleeding points coagulated with bipolar diathermy.

\section{Transvaginal hydrolaparoscopy}

Transvaginal hydrolaparoscopy was introduced in order to eliminate the risks of general anaesthetic at laparoscopy while maintaining a high diagnostic yield [6]. The procedure is done on an outpatient basis under local anaesthetic. A small diameter $(2.7-2.9 \mathrm{~mm})$ endoscope is inserted through the posterior vaginal fornix into the pouch of Douglas, using a blunt dilating trocar. Saline or ringer lactate infusion distends the peritoneal cavity. A panoramic view of the lower pelvis is obtained along with a more physiological view of the tubo-ovarian relationship. Diluted methylene blue dye can be injected transcervically and the fimbria visualised to demonstrate tubal patency. The transvaginal laparoscope can also be directed through the fimbrial end of the fallopian tube to act as a salpingoscope. The inability to perform surgery during transvaginal hydrolaparoscopy has been overcome by use of larger-diameter trocars with operative channels; however, only minimal adhesiolysis or a biopsy can be performed. Inability to visualise the anterior pelvis or the upper abdomen are other disadvantages.

\section{Falloposcopy/salpingoscopy}

Falloposcopy is a transvaginal microendoscopic technique to explore the human Fallopian tube from the uterotubal ostium to the fimbrial end. It enables visualisation of endotubal disease. The instrument consists of a guide wire and a catheter over the wire that has been specifically designed to explore the human fallopian tube. This is used in conjunction with hysteroscopy. Upon visualisation of the tubal ostium, the hysteroscope is fixed in position. The tube is cannulated as completely as possible via the working channel of the hysteroscope .The catheter and guide wire is threaded through the entire length of the fallopian tube. This manoeuvre is aided by continuous distension of the tubal lumen by a pump at 5$15 \mathrm{ml} / \mathrm{min}$. Once the catheterisation is complete, the wire is withdrawn and a falloposcope with 3000 pixels is inserted. The tubal lumen is now visualised in a retrograde fashion. Due to the intense light in close proximity of tissues, "white out" occurs commonly. Kinking occurs 
frequently. This leads to damage to the catheter and impedes successful falloposcope insertion. Lastly, if the endometrium is in the advanced proliferative phase, it may be difficult to visualise the ostia.

Falloposcopy is based on the principles of salpingoscopy. Salpingoscopy is performed during laparoscopy and only examines the ampullary mucosa. Its role in diagnosis and management in ART is therefore limited.

\section{Hysteroscopy}

Hysteroscopy has come a long way from its conception about 100 years ago. The hysteroscope has become narrower and the optics advanced. There is a shift from performing diagnostic hysteroscopy under general anaesthetic to an outpatient approach under local anaesthetic. Use of 2.5- to $3.5-\mathrm{mm}$ hysteroscopes allows adequate visualisation of the uterine cavity. The Versascope is the latest edition of thin hysteroscopes to be used on an outpatient basis. It has the advantage of incorporating an operating channel within its outer sheath. Small polyps can be removed or an endometrial biopsy performed using a biopsy forcep inserted through the operating channel.

It is debatable whether hysteroscopy should be used as a first choice in evaluating the uterine cavity in ART. Hysterosalpingography (HSG) is a relatively inexpensive procedure that provides important information about the endocervical canal, the region of the internal os, the uterine cavity and the entire courses of the fallopian tubes. For the infertile patient, the latter information is invaluable. Furthermore, this information could alter the patient's management. For example, findings of large hydrosalpinges not amenable to corrective surgery would prompt an early referral for in vitro fertilisation and embryo transfer; however, hysteroscopy provides more detailed information of the uterine cavity. It allows direct visualisation of the endometrial cavity, the lesions can be mapped accurately and intrauterine surgery can be performed at the same time.

\section{Resection of polyps/myoma}

It is debatable as to how small endometrial polyps or submucous myoma lead to infertility. They may, however, more commonly lead to pregnancy wastage. Presence of endometrial polyps/myoma is usually noticed at ultrasound scan either at investigation or treatment of infertility. Polyps are resected without damage to the surrounding endometrium (Table 3). The feasibility of resection of myomas depends upon their intramural component. When $50 \%$ of the myoma is intramural, attempts to resect may lead to haemorrhage from the myometrial bed. It is therefore best enucleated by conventional myomectomy. Myomas that are pedunculated are removed after transection their stalks. Another easier approach is avulsing the pedunculated myoma with a polyp forcep and coagulating any bleeders at the base. It is not necessary to perform
Table 3 Types of hysteroscopic surgery in ART

Resection of polyps

Resection of myoma(s)

Division of intrauterine adhesion

Division of intrauterine septum

simultaneous laparoscopy routinely while resecting a sessile myoma. The surgery is usually done during the follicular phase of the menstrual cycle. After inspecting the endometrial cavity, any blood vessels on the surface are coagulated. The myoma is then "shaved" down to a point even with the plane of the adjacent endometrium or slightly below. In the short term, recurrence rate is low. If myomas on opposite uterine walls are resected, opposing surfaces may develop adhesions. In order to reduce the incidence of postoperative intrauterine adhesions, it may be advisable to treat one surface initially and the other 1 month later after the first operative site has healed. Another option is insertion of an intrauterine device to prevent readherence. Conjugated equine estrogen $1.25 \mathrm{mg}$ daily is prescribed for 25 days and on the last 5 days of the estrogen therapy, $10 \mathrm{mg}$ of medroxyprogesterone acetate is prescribed. Prior to any form of ART, the endometrial cavity is inspected to assess its contour and to verify that adhesions have not occurred. The main advantage of hysteroscopic resection in patients who subsequently conceive is that they may be allowed to labour normally and undergo a caesarean section for obstetric reasons only as compared with women with routine myomectomy.

\section{Division of intrauterine adhesions}

Intrauterine adhesions (IUA) can lead to hypomenorrhoea, pregnancy wastage (including first- and secondtrimester miscarriage), errors of placental implantation (placenta accreta, increta and percreta) and infertility. Prior to the advent of hysteroscopy, diagnosis of intrauterine adhesions (IUA) was based on history of hypomenorrhoea and HSG findings of single or multiple irregular filling defects; however, the diagnosis can only be made with certainty only when there is direct inspection of the uterine cavity. Once the diagnosis is made, the aim of treatment is restoration of uterine architecture to normal, to prevent readherence of the uterine walls, to provide stimulation for endometrial growth over the freshly dissected surfaces and to verify that the uterine cavity is normal prior to any attempts at ART. Insertion of the hysteroscope should be under direct vision. Attempts to identify normal landmarks, such as the tubal ostia, are made initially. The extent and thickness of the IUA are first noted. If no landmarks are identified and if the hysteroscope cannot be advanced safely, laparoscopy is performed before further hysteroscopic efforts are made. Once the pelvis is inspected, the light intensity for the laparoscope is reduced markedly. Hysteroscopic division is now resumed. If the hysteroscopic lysis is advertently carried into the myometrium, the light from the hyster- 
oscope will shine brightly through the uterine serosa. Hysteroscopic dissection should then be redirected in order to prevent uterine perforation. Miniature scissors are used to divide adhesions under direct vision. An inert intrauterine device is inserted into the cavity in order to prevent readherence. Inert devices, such as the Lippes loop, are preferred as they have a larger surface area than the common T-shape IUD and cause less intrauterine reaction than those which release copper. Another option is inflation of the balloon of a 10-F Foley catheter in the endometrial cavity. This is retained for 5-7 days. All patients are prescribed conjugated estrogen $2.5 \mathrm{mg}$ twice daily for 60 consecutive days. Progesterone withdrawal bleeding is induced from day 56 to 60 and the IUD removed. An outpatient hysteroscopy should then be performed to rule out readherence.

\section{Division of intrauterine septum}

Reproductive failure is the most common symptom in patients with congenital uterine anomalies. These defects cause infertility occasionally. A complete infertility investigation is mandatory before surgical intervention for a uterine anomaly. Women with intrauterine septum do not require investigation of the urinary tract. Prior to planning hysteroscopic therapy, laparoscopy is required to rule out bicornuate uterus. Once the diagnosis is established, miniature scissors are used to divide the septum. For septums $<3 \mathrm{~cm}$ in width, the division is carried cephalad from the most inferior point of the septum and directed laterally at the fundus. For septums $>3 \mathrm{~cm}$ at the fundus a different approach is needed. The dissection starts at the most inferior point of the septum. It is then carried out along the lateral margin of the septum until $0.5 \mathrm{~cm}$ from the junction of the myometrium. The other lateral margin is incised alternately until the original very broad Vshaped septum is converted into a broad notch at the fundus. This technique allows minimal bleeding and impeccable views. Dissection is now carried out between the tubal ostia to restore anatomy as normal as possible. It is important to maintain traction on the cervix throughout the procedure. This will maintain the uterus in an axial plane and would prevent anterior or posterior uterine wall perforation. Postoperative management is similar to that described for resection of submucosal myomas.

\section{Results}

Laparoscopy is a commonly performed endoscopic procedure in the evaluation and management of the infertile couple. The economic and safety issues of diagnostic laparoscopy should be considered. In a large study spanning 8 years and 29,966 diagnostic and operative laparoscopies, the complication rate of diagnostic laparoscopy was 1.84 of 1000 and 4.30 of 1000 procedures for major laparoscopic surgery [7]. The rate of complications treated by laparotomy for diagnostic and major laparo- scopic surgery was 1.51 per 1000 and 3.76 per 1000 , respectively. One-third of complications $(34.1 \%$ were secondary to creation of the pneumoperitoneum or installation of the trocars). This rate was $13.1 \%$ for urological complications, $32.5 \%$ for bowel complications and $48.9 \%$ for haemorrhagic complications. This prompts the statement that diagnostic laparoscopy is not an innocuous procedure, and although the risk is lower than with operative laparoscopy [8], it nonetheless exists [7, 9, 10]. Given that laparoscopy carries a certain risk along with the complications of general anaesthesia, the question arises as to whether it should be performed in women without any risk factors for evaluation of infertility. The HSG was found to be accurate with only $65 \%$ sensitivity in diagnosing tubal patency as compared with laparoscopy [11]. It was even more unreliable in detecting peritubal adhesions. In infertile couples laparoscopy reveals abnormal findings in $21-68 \%$ of the cases after normal HSG $[12,13,14,15,16,17,18]$. In a recent study, due to abnormal findings at laparoscopy, $25 \%$ of women undergoing stimulated intrauterine insemination had a different treatment [19]; thus, evidence suggests that laparoscopy has a role to play in evaluating women with unexplained infertility prior to ART.

Clinical guidelines published by the Royal College of Obstetricians and Gynaecologists suggest that surgical ablation of minimal and mild endometriosis improves fertility. This is also confirmed by a recent meta-analysis [20]. Surgical treatment of mild endometriosis has been found to be more cost-effective and with better outcomes than stimulated IVF [21]; however, Taylor and Olive [22] calculated that based on data derived from the Canadian Collaborative Group of Endometriosis [23], 33.5 laparoscopies would need to be performed in patients with an otherwise normal infertility evaluation to achieve one ongoing pregnancy for surgical ablation of early stage endometriosis. In a prospective study, 39 women with endometriomas were treated by fenestration and ablation of the capsule by lasers or bipolar diathermy. Although the cumulative pregnancy rate was $39.5 \%$, suggesting that fenestration and capsule ablation of endometriomas is an effective treatment [24], in units with a higher than average success rate at IVF, surgery for moderate to severe endometriosis is less cost-effective than stimulated IVF [21].

Very few studies have been undertaken to explore the effects of laparoscopic adhesiolysis on fecundity. Open adhesiolysis has been shown to increase spontaneous pregnancy rates [25]. The cumulative pregnancy rates were higher at 12 and 24 months in the group that was treated as compared with those who were untreated. Similar effect of laparoscopic adhesiolysis on fecundity has been proposed recently [19]. Diagnosis and treatment of adhesions at laparoscopy has been shown to be most cost-effective measure when comparing IVF with tubal surgery [26]. No statistically different cumulative conception rates were found between microsurgical techniques and laparoscopic adhesiolysis [27]. Since IVF has developed and success rates have improved, there is a 
debate as to the place of tubal reconstructive surgery. Only patients with mild to moderate tubal damage should be considered for tubal reconstructive surgery while all others should be considered for IVF [21, 28]. Obviously this would depend on local availability and the success rates of the unit.

Leiomyomas represent an increasing medical problem in women attempting to conceive at a more advanced age. Submucosal myomas are treated by standard operative hysteroscopy. Several studies have demonstrated successful reproductive outcome after hysteroscopic removal of submucosal myomas in infertile women $[29,30,31,32$, 33 . In a recent study, the pregnancy rate was significantly better when myoma was the exclusive aetiology of infertility (41.6\%), and when the lesion was $\geq 50 \mathrm{~mm}$ in size $(57.1 \%)$ [34]. Studies on the effect of intrauterine adhesiolysis or division of intrauterine septum are few and small. Meta-analysis of published retrospective data comparing pregnancy outcome before and after hysteroscopic septoplasty indicate a marked improvement after surgery [35]. The pregnancy rate in 116 patients following the operative hysteroscopy for an intrauterine septum in a prospective follow-up period of 24 months was 50\%: 44 patients $(74.6 \%)$ had term deliveries; $11(18.6 \%)$ had first trimester miscarriage; and $4(6.8 \%)$ had preterm delivery [36].

\section{Future}

Infertility affects one in seven couples in the UK. Although the prevalence does not seem to have increases, many more couples are seeking help than previously. A typical district general hospital in the UK may see around 230 new consultant referrals each year. Common gynaecological conditions, such as abnormal uterine bleeding, are investigated in a simple one-stop menstrual problem clinic. Randomised trials have shown that this approach is efficient, cost-effective and results in increased patient satisfaction [37]. In contrast, infertility investigations and treatment are a long process. General practioners are the first step of the ladder for a couple with infertility seeking help. After baseline investigations, they are referred to secondary or tertiary centres for treatment. This is timeconsuming and puts additional stress on the couple during this waiting period. Couples could wait up to a year to find out what type of treatment would be required to achieve a pregnancy. Recently, a one-stop approach has been proposed for couples presenting with infertility [38]. Transvaginal hydrolaparoscopy is systematically combined with mini-hysteroscopy, chrompertubation, fimbrioscopy and sapingoscopy, if needed. The combination of these procedures is referred to as "fertiloscopy" [39]. This approach has been shown to be feasible [39, 40, 41, $42,43]$, accurate for determining tubal patency $(95 \%)$ [44], safer than laparoscopy with respect to bowel injuries [45], better tolerated [43, 44, 46] and certainly costeffective $[43,46]$. One must be wary that the one-stop approach for infertility investigations is appropriate for patients without obvious pelvic pathology; therefore, a detailed history, gynaecological examination and transvaginal sonography should be used to exclude patients with vaginal infection, obliteration of pouch of Douglas, fixed retro-verted uterus, lateral development of the cervix or suspected pelvic tumour [38]. One drawback of THL is the inability to examine the anterior pelvis, upper abdomen and perform operative procedures such as ovarian drilling, ablating superficial endometriosis, adhesiolysis or treatment of ovarian endometriomas; however, with more advance in technology and instrumentation, these drawbacks could be overcome in the future.

\section{References}

1. Surrey ES (2000) Endoscopy in the evaluation of the women experiencing infertility. Clin Obstet Gynecol 43:889-896

2. Quint EH, Wang FL, Hurd WW (1996) Laproscopic transillumination for the location of anterior abdominal wall blood vessels. J Laparoendosc Surg 6:167-169

3. Lundorff P,Hahlin M, Kallfelt B et al. (1991) Adhesion formation after laparoscopic surgery in tubal pregnancy: a randomized trial versus laparotomy. Fertil Steril 55:700-704

4. Luciano AA (1992) Management of adhesions via laparoscopy in operative laparoscopy. McGraw-Hill, New York, pp 121126

5. Johnson NP, Mak W, Sowter MC (2002) Laparoscopic salpingectomy for women with hydrosalpinges enhances the success of IVF: a Cochrane review. Hum Reprod 17:543-548

6. Gordts S, Campo R, Rombauts L et al. (1998) Transvaginal hydrolaparoscopy as an outpatient procedure for infertility investigation. Hum Reprod 13:99-103

7. Chapron C, Querleu D, Bruhat MA et al. (1998) Surgical complications of diagnostic and operative gynaecological laparoscopy: a series of 29996 cases. Hum Reprod13:867-872

8. Harkki-Siren P, Kurki T (1997) A nationwide analysis of laparoscopic complications. Obstet Gynecol 69:373-384

9. Jansen FJ, Kapiteyn K, Trimbos-Kemper T et al. (1997) Complications of laparoscopy: a prospective multicentre observational study. Br J Obstet Gynaecol 104:595-560

10. Querleu D, Chevalier L, Chapron C, Bruhat MA (1993) Complications of gynecological laparoscopic surgery. A French multicentre collaborative study. Gynecol Endosc 2:3-6

11. Mol BWJ, Collins JA, Burrows EA et al. (1999) Comparison of Hysterosalpingography and laparoscopy in predicting fertility outcome. Hum Reprod 14:1237-1242

12. Wood GP (1983) Laparoscopic examination of the normal infertile woman. Obstet Gynecol 62:642-643

13. Henig I, Prough SG, Cheatwood M, DeLong (1991) Hysterosalpingography, laparoscopy and hysteroscopy in infertility. A comparative study. J Reprod Med 36:573-575

14. Opsahl MS, Miller B, Klein TA (1993) The predictive value of hysterosalpingography for tubal and peritoneal infertility factors. Fertil Steril 60:444-448

15. Cundiff G, Carr BR, Marshburn PB (1995) Infertile couples with a normal hysterosalpingogram. Reproductive outcome and its relationship to clinical and laparoscopic findings. J Reprod Med 40:19-24

16. Belisle S, CollinsJA, Burrows EA, Willan AR (1996) The value of laparoscopy among infertile women with tubal patency. J Soc Obstet Gynecol Can 18:326-336

17. al Badawi IA, Fluker MR, Bebbington MW (1999) Diagnostic laparoscopy in infertile women with normal hysterosalpingograms. J Reprod Med 44:953-957

18. Corson SL, Cheng A, Gutmann JN (2000) Laparoscopy in the "normal" infertile patient: a question revisited. J Am Assoc Gynecol Laparosc 7:317-324 
19. Sandra J, Tanahatoe S, Hompes GA, Lambalk CB (2003) Investigation of the infertile couple: Should diagnostic laparoscopy be performed in the infertility work up programme in patients undergoing intrauterine insemination? Hum Reprod 18:8-11

20. Olive DL, Pritts EA (2002) The treatment of endometriosis: a review of the evidence. Ann N Y Acad Sci 955:360-372

21. Philips Z, Bazarraza-Llorens M, Posnett J (2000) Evaluation of the relative cost-effectiveness of treatments for infertility in the UK. 15:95-106

22. Taylor HS, Olive DL (197) Unexplained infertility: the role of laparoscopy. Infertil Reprod Med Clin North Am 8:603-609

23. Marcoux S, Maheux R, Berube S (1997) Laparoscopic surgery in infertile women with minimal or mild endometriosis: Canadian Collaborative Group on Endometriosis. N Engl J Med 337:217-222

24. Jones KD, Sutton CJG (2002) Pregnancy rates following ablative laparoscopic surgery for endometriomas. Hum Reprod 17:782-785

25. Tulandi T, Collins JA, Burrows E, Jarrell JF, McInnes RA, Wrixon W, Simpson CW (1990) Treatment-dependent and treatment-independent pregnancy among women with periadnexal adhesions. Am J Obstet Gynecol 162:354-357

26. Khare VK, Consonni R, Martin DC, Winfield AC (1995) Use of algorithmic pathways to develop quality, cost-effective clinical care. J Am Assoc Gynecol Laparosc 2:169-174

27. Saravelos HG, Li TC, Cooke ID (1995) An analysis of the outcome of microsurgical and laparoscopic adhesiolysis for infertility. Hum Reprod 10:2887-2894

28. Standell A (2003) Tubal infertility Hum. Reprod 18:85xviii86xviii

29. Donnez J, Gillerot S, Bourgonjon D, Clerckx F, Nisolle M (1990) Neodymium:YAG laser hysteroscopy in large submucous fibroids. Fertil Steril 54:999-1003

30. Goldenberg M, Sivan E, Sharabi Z, Bider D, Rabinovici J, Seidman DS (1995) Outcome of hysteroscopic resection of submucous myoma for infertility. Fertil Steril 64:714-716

31. Giatras K, Berkeley AS, Noyes N, Licciardi F, Lolis D, Grifo JA (1999) Fertility after hysteroscopic resection of submucous myomas. J Am Assoc Gynecol Laparose 6:155-158

32. Varasteh NN, Neuwirth RS, Levin B, Keltz MD (1999) Pregnancy rates after hysteroscopic polypectomy and myomectomy in infertile women. Obstet Gynecol 94:168-171

33. Vercellini P, Zaina B, Yaylayan L, Pisacreta A, Giorgi O de, Crosignani PG (1999) Hysteroscopic myomectomy: long-term effects on menstrual pattern and fertility. Obstet Gynecol 94:341-347

34. Fernandez H, Sefrioui O, Virelizier C, Gervaise A, Gomel V, Frydman R (2001) Hysteroscopic resection of submucosal myomas in patients with infertility. Hum Reprod 16:1489-1492

35. Hayden AH, Li TC, Cooke ID (2000) The septate uterus: a review of management and reproductive outcome. Fertil Steril 73:1-14

36. Kupesic S, Kurjak A (1998) Diagnosis and treatment outcome of the septate uterus. Croat Med J 39:185-190

37. Kremer C, Duffy S, Moroney M (2000) Patient satisfaction with outpatient hysteroscopy versus day case hysteroscopy: randomised controlled trial. Br Med J 320:279-282

38. Gordts S, Campo R, Puttemans P, Verhoeven H, Gianaroli L, Brosens J, Brosens I (2002) Investigation of the infertile couple : a one-stop outpatient endoscopy-based approach. Hum Reprod 17:1684-1687

39. Watrelot AA, Dreyfus JM, Andine JP (1999) Evaluation of the performance of fertiloscopy in 160 consecutive infertile patients with no obvious pathology. Hum Reprod 14:707-711

40. Bajzak KI, Winer WK, Lyons TL (2000) Transvaginal hydrolaparoscopy, a new technique for pelvic assessment. J Am Assoc Gynecol Laparosc 7:562-565

41. Darai E, Dessolle L, Lecuru F, Soriano D (2000) Transvaginal hydrolaparoscopy compared with laparoscopy for the evaluation of infertile women: a prospective comparative blind study. Hum Reprod 15:2379-2382

42. Dechaud H, Ali Ahmed SA, Aligier N, Vergnes C, Hedon B (2001) Does transvaginal hydrolaparoscopy render standard diagnostic laparoscopy obsolete for unexplained infertility investigation? Eur J Obstet Gynecol Reprod Med 94:97-102

43. Moore ML, Cohen M (2001) Diagnostic and operative transvaginal hydrolaparoscopy for infertility and pelvic pain. J Am Assoc Gynecol Laparosc 8:393-397

44. Cicinelli E, Matteo M, Causio F, Schonauer LM, Pinto V, Galantino P (2001) Tolerability of the mini-pan-endoscopic approach (transvaginal hydrolaparoscopy and minihysteroscopy) versus hysterosalpingography in an outpatient infertility investigation. Fertil Steril 76:1048-1051

45. Gordts S, Watrelot A, Campo R, Brosens I (2001) Risk and outcome of bowel injury during transvaginal pelvic endoscopy. Fertil Steril 76:1238-1241

46. Gordts S, Campo R, Brosens I (2000) Office transvaginal hydrolaparoscopy for early diagnosis of pelvic endometriosis and adhesions. J Am Assoc Gynecol Laparosc 7:45-49 